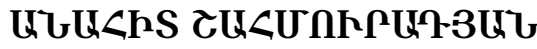

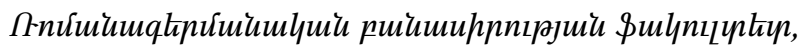

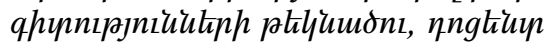

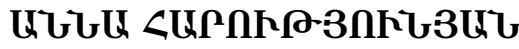

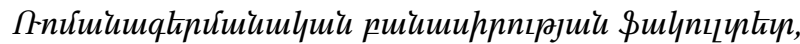

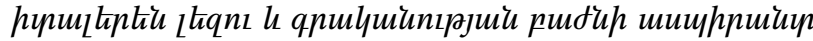

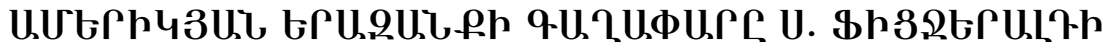

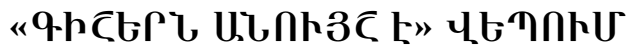

«922tinu uunts2 5» \$hgetinumphh

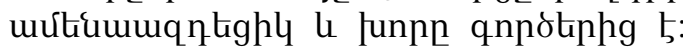
hp htennuhu' rhphi, elqunuqpniu b ununuinulnn, bukjugh $\mathrm{l}$ lh hnqunp

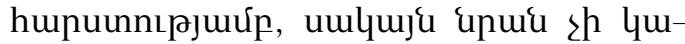

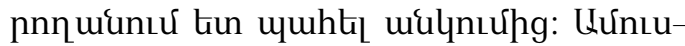

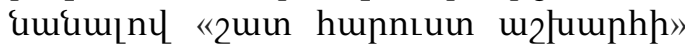
utplyujugnıgsh htin' Thph hnqunn w2-

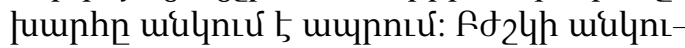
un, nnptinqnipjnıun - nnptinqnıpjnı́ 5

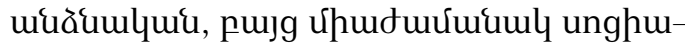

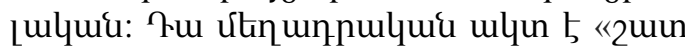

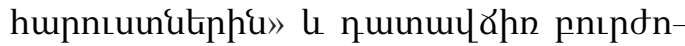
เulqui Lứtphlqujh hưưn, nnp unuiug

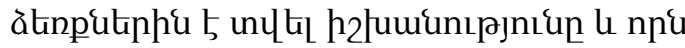

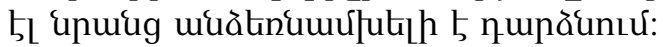

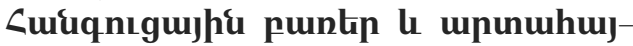

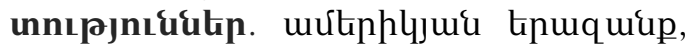

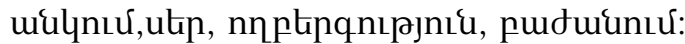

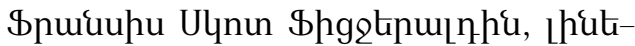

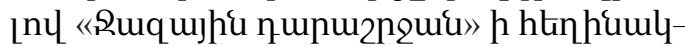

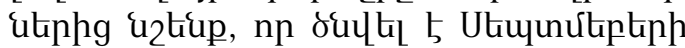
24-hu, 1896 p-hu, hnцuinulyui qupn-

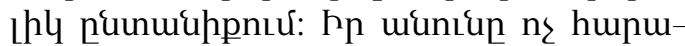

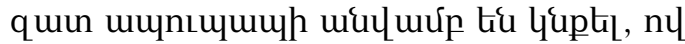

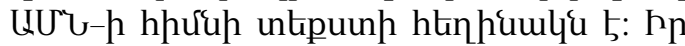

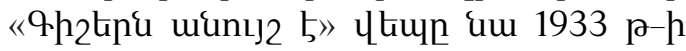
ш2

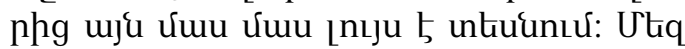
yunnn 5 plü, np ujuunten sluwu ungh-

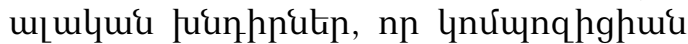
ứpnngulqui 25: Fujg, hhunlt, ¿tiup

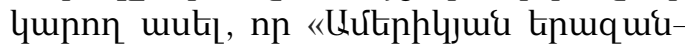

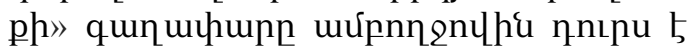

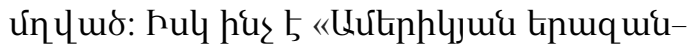

pL»:

Uju ununwhujunnıpjnıun hwơwu oq-

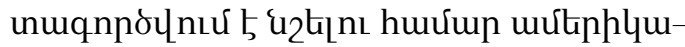
ghutiph ljuuph hnteu[utinn, hu<utiu ujno-

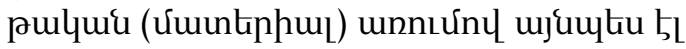
hnqunn: «Uútnpljuwu tipuquipp» hum-

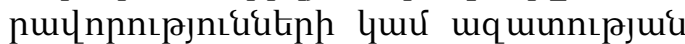
hntuu[u b:

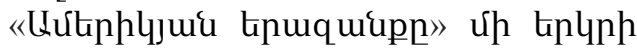
úuuhu 5 , nnukin jnıpupueşnın úmpnnı

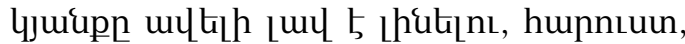

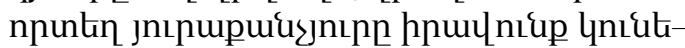

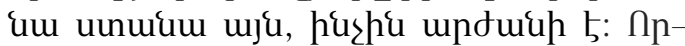

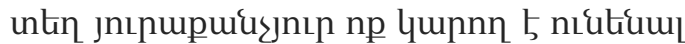
uju, hus guulquintú b, Gunnn 5 àtinp phipta mja, husn tiplum dưumuml wuhuuwutilh 5 tnkt hptú (J. Adams 1931, 6):

«9.22tinu wunıs2 5» \$hgetinuinhh

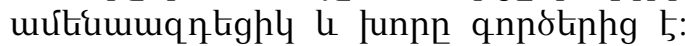

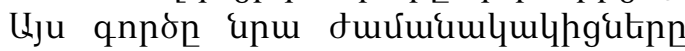

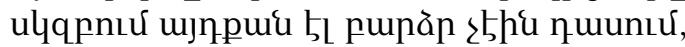

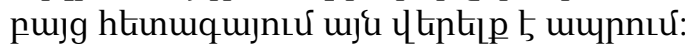
પtuuh qnnonnnıpjntuutinn, (thot hu2lh

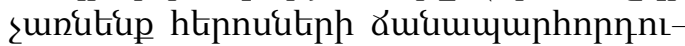

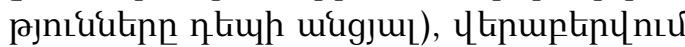
5 20-mquukinh 2 ltuhs, ujuhupd'«Ru-

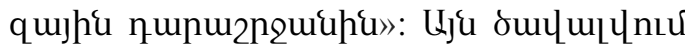
5 \$nuuuhujnıर्u, đuthghuphujnıu, hunu-

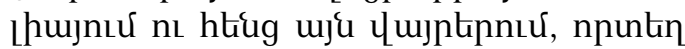

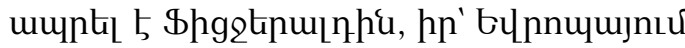
qunultans unuphutiphe: Cnqtipurumlyue

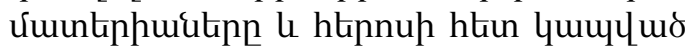
pninn úuuumqhumulue qnoóntutint-

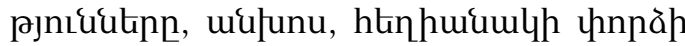

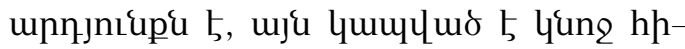




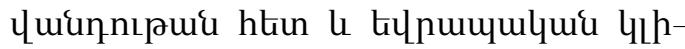
uplumutinnıu pntonsưutinh' đutgounhu-

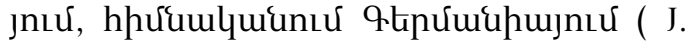
Chamberlain 1934, 2):

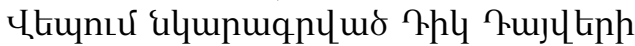

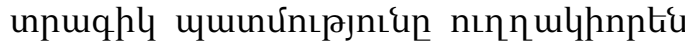

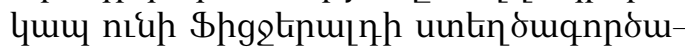
quu aulquinuqph le nunn junhtiph htiu:

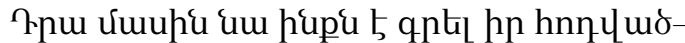

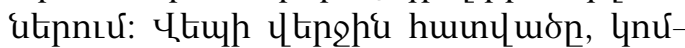
unqhghnu le qunnıgumópujhu unnıư nnl, hnulquimumbu yhumu 5 , ptiph

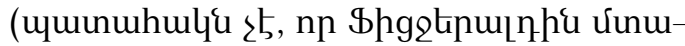

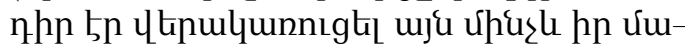
hn, le ulqukl th unntiu, umlquju shuugntig (thequguti):

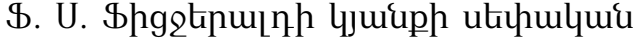

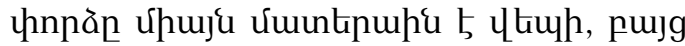
n乞 pte npu hưuuun:

«912kinu uunts2 5 " puqúulynnưuup,

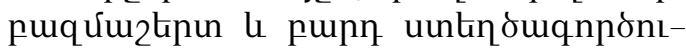

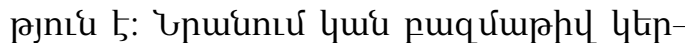
umunutin: Gnughg úp puupun uunnıu

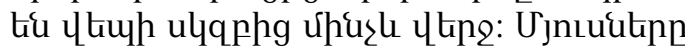
hujunuy nıu tú buhq nnujhu, ujunshtinl

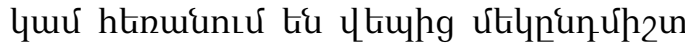

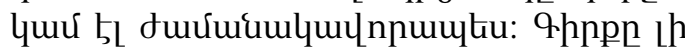
5 ujnıdtunushu úuuknnu, tenptưu htinhwiumlh Gumbuignu:

Gtpumunutinh u hpununānıpjnıưutinh

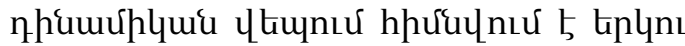

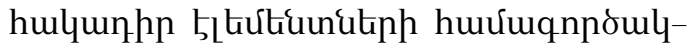
gnıpjuúp, úhưjuuhghg uwhưuumquin-

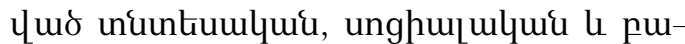
nnjulqui huulpnıpjnıuutinnl: Rpuiahg

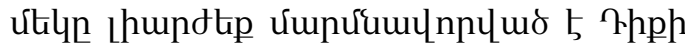

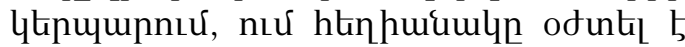

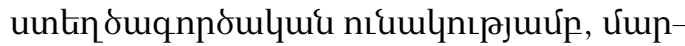

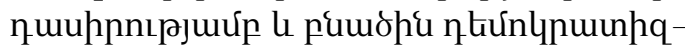
unl: Ujnıun unnté ulqunuqnц

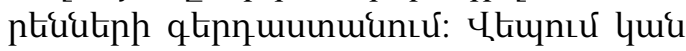
uJl hkpnuukn unıjumbu, pujg upuép

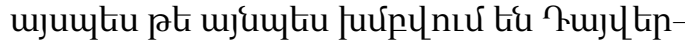
utiph lu nınptiưtiph 2nınen: nınptưutinn hptiug 2nquumuunnư úto lu guplinn ntip

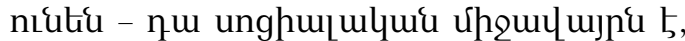
npukn lu htipnu, le úhudưưuuml nıd, nnp lnnowưnıu 5 unwu: 9pph ujnıdtu

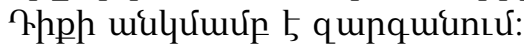

nınptiuutinn «Zuun hupnsuun úunnhl

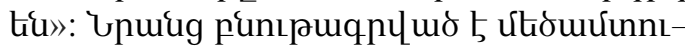

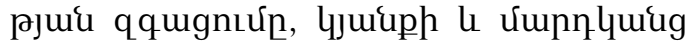

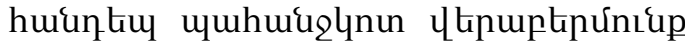

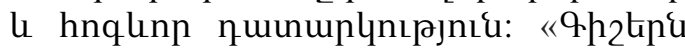
uunts2 5 » ukplqujugunıu 5 qtnupltiu-

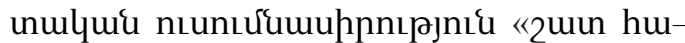
nnıuun úupn thu unuiug w2lumphh pupnjulquinıpjui

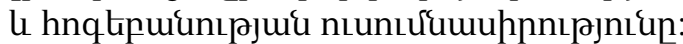

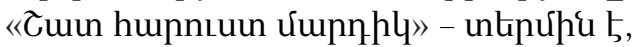

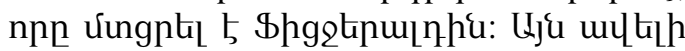
juju hưwuun niuh, puu nınhn hưuunn:

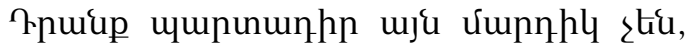

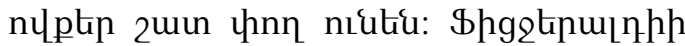
htipnuutinh úte qui ujuupuutinn, nu-

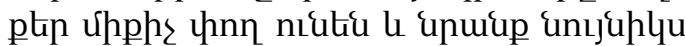

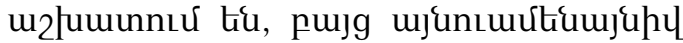

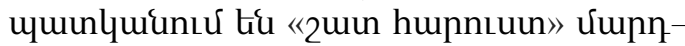
yuiug luwlhu ( Р. Льıсцев, 2008, 3):

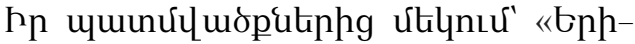

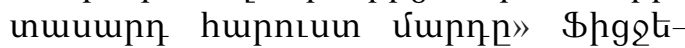
nuinhiu uunıu $b$, np hupntuunutinn úta

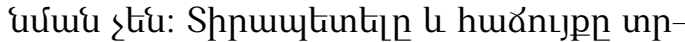
ц ư tú unuiag, lu npuing unuiug únu

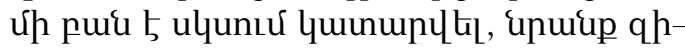

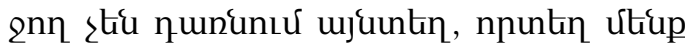

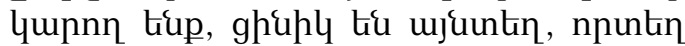

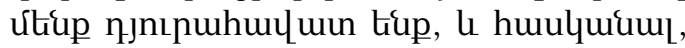

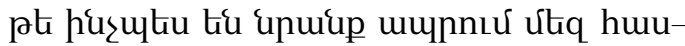

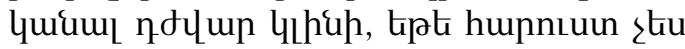

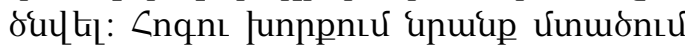
ku, np úkquhg juqu tú: Unıjupul tipt

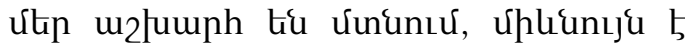
upuúp únmơnıú tú, np úkquhg juýu tú,

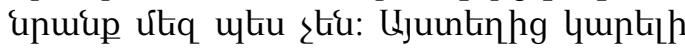
5 tqpulugukt, np «hunnıuu ưnnn»unghur-hnqtpuiumlyue unhư 5 , npnup unuquuntú tí tiplns tiptip utinntungut-

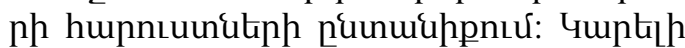
5 uukl, np \$hgetpulnhh "2uun hupnıuun umpnhl»- dunuuqualyu unhuunnlnu-

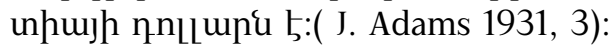

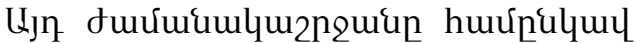

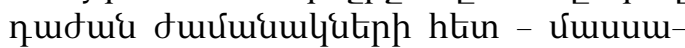




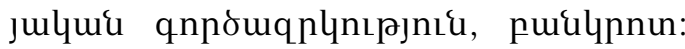

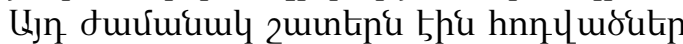

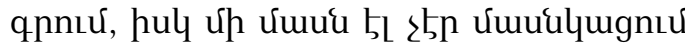

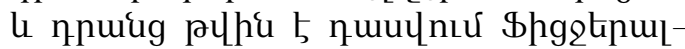

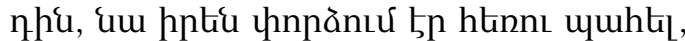

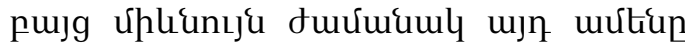

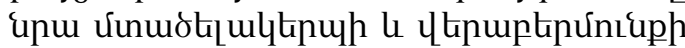
ไnu 5 uqnnเư:

nınptiuutinn 2uun hupnıuu ưunn-

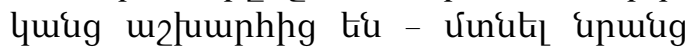

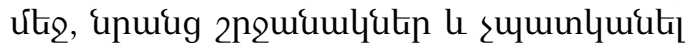

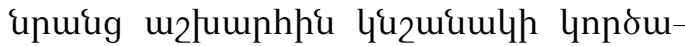
unıu, husuku Thph únu tnuu:

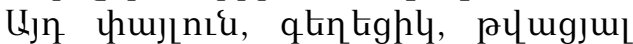

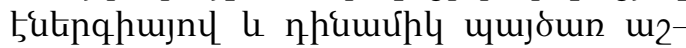
jumphn, jumpnsuhl 5 , hpulquinıu huc-

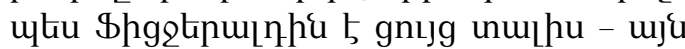
unpuin 5 , wupnıdth hhluin: ঢnuip n々hur ¿ntuku pugh thnnhg: Unuug puntpuqpnıu 5 Lhnuhu hnqunph pugu-

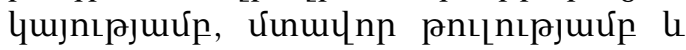

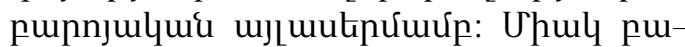

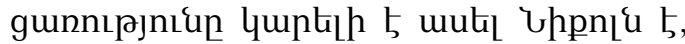

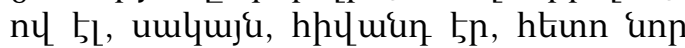
upuug hul wumpltig, tipp upntiu hupp pnıdy tig, hul Rhpn lnnowiul tig: nınptiu-

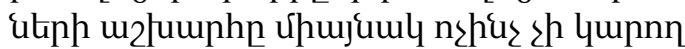
uukt, unıjuhul nıpu|umumi:

Fdh24 Rusltinn - qnulhs htunupnphp 4tinume 5: \$hgetnuinhe unue juilpnl, jumphqưunhl, ununuinuulnn,

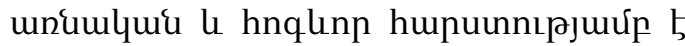
ulumuqnntu: funtjpny \&u ưnuonn, qh-

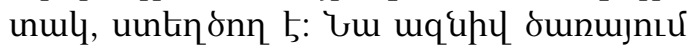
5 umpnlyuig le puntpjuin: Cnqtipuint-

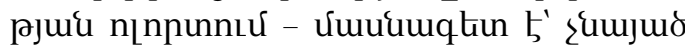
tphunuump unuphph upntes huugnti $b$ àtinp ptiptal htinhumlnnıjnıu qhunulumu

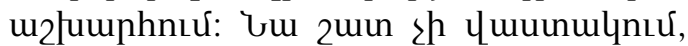

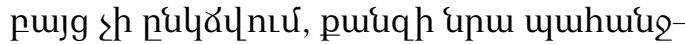
utipe $b_{L}$ hứtiuu ted: Gnu úhunpn qpun-

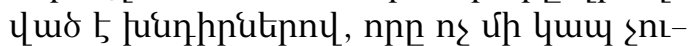
uh thnnh htiu:

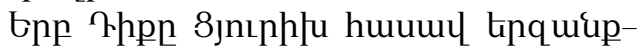

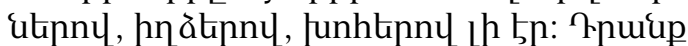

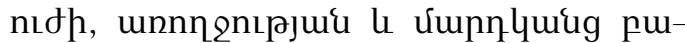

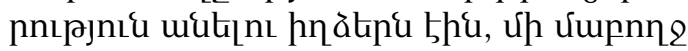

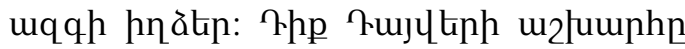

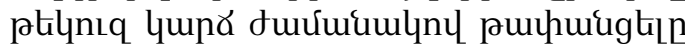

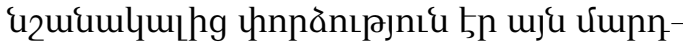

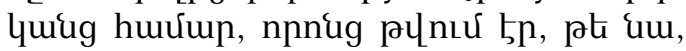
hu24h unutind jnıpupuusjnıph undu-

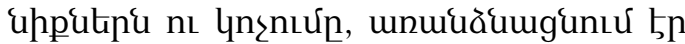

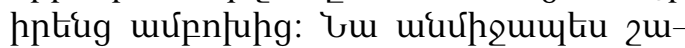

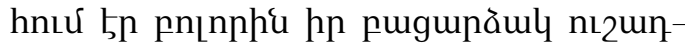

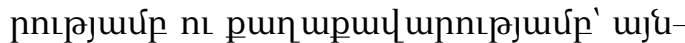

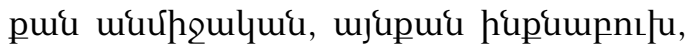

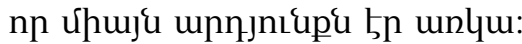

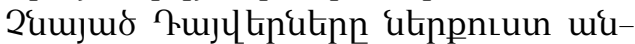
unupptin thu w2tumphly ljuiph optiup-

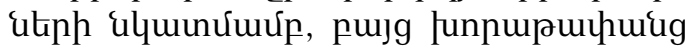
thukind' 25hu funıumhntú unu dưư-

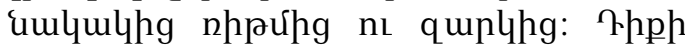

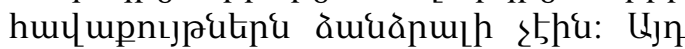

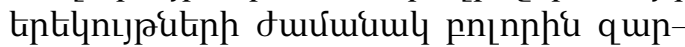

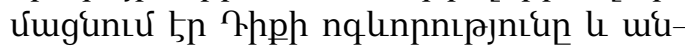
àunınugnıpjnıun, husutu uwa upu

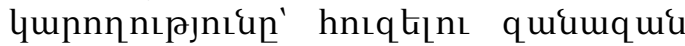
úmpntuug, npnughg jnıpupuusjnınр

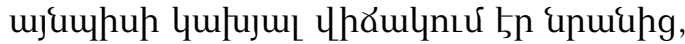

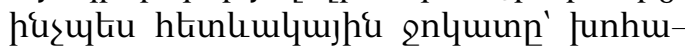

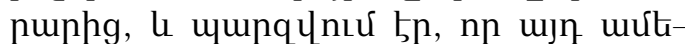

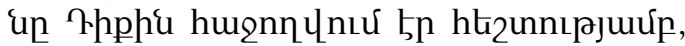
unwug ahqh, lu np Thpp ptinluu nicutp

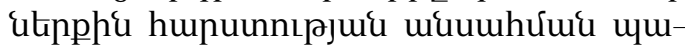
zunitip jnınupuidsnıph hưưu, nц hn 4unppn 4qqun:

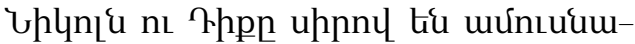

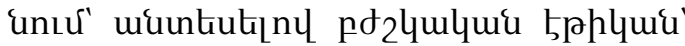

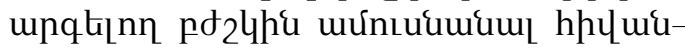

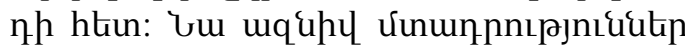

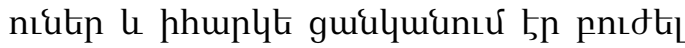

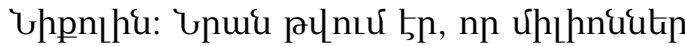

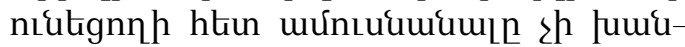

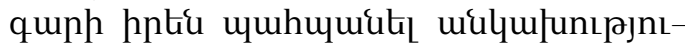
up, 2unntumbli umudumqhunulume qnp-

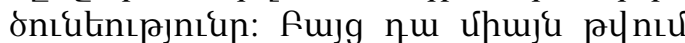

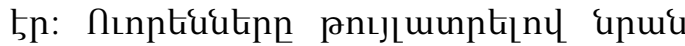

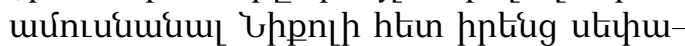

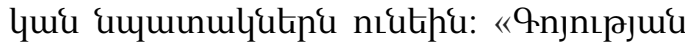
hưự hnp ún wơ umupunp' thlin tgum-

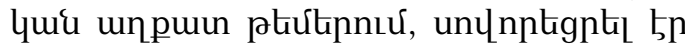

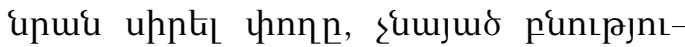

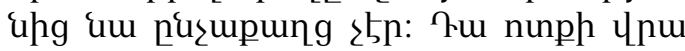




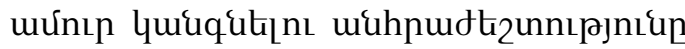
25n. pusg lo ujuutiu tipptip uınpuiu hupumuluoum $25 p$ tngla, tipptip hptiu ujn-

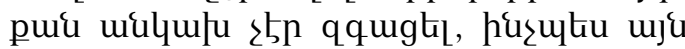

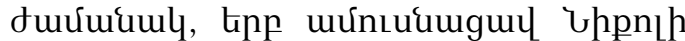

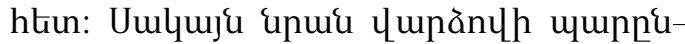
lne ưưu lnul unltghiu, lu hupp hus-np liknu pntJl unltgg, np hp nng qhumiungn

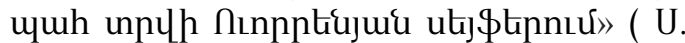
\$hgqtinuin 2015, 153):

unuip, ujumku uumg qukghu Ruj-

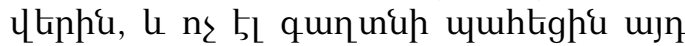

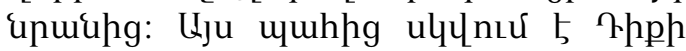

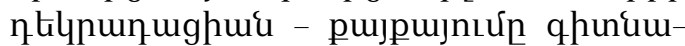

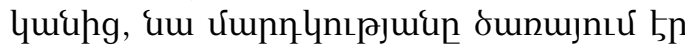

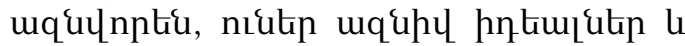

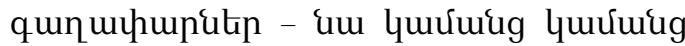

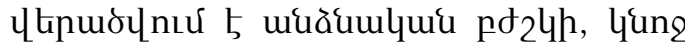

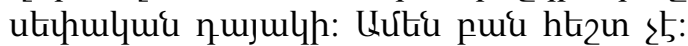

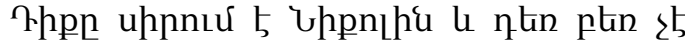

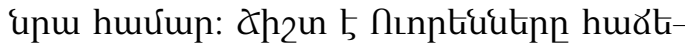

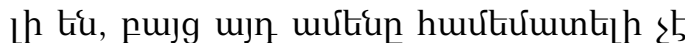

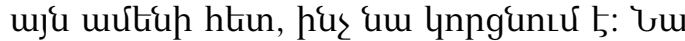
ynnguntu $5 \mathrm{hp}$ ununuunn, umueumq

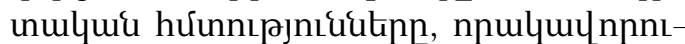
un, hnqtiluu butinqhuid, upurh únuorn-

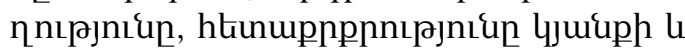
ưmnluwug huuntum (P. Льссиев 2008, 2):

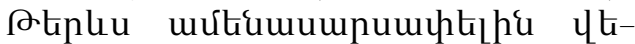
nuóưn

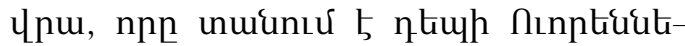
nh w2lumphn' ulqqpnıu wuàh hınıqnứ,

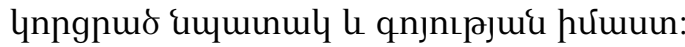
Tuh 5 quihu, tipp um uJlluu ukinp 25 uhpnihu:

fd्2lh nnptinqnıpjnıนn, wulynıún -

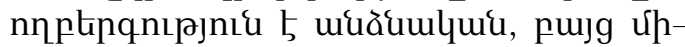

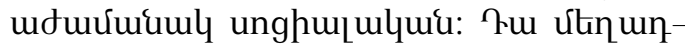

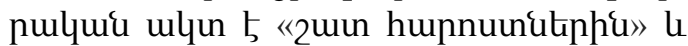

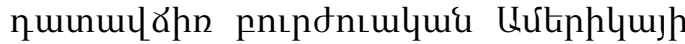
hưưn, nupten uuảtinumúuluth tu nupàtıl h2luuunıpjư zunphhu:

qpph ulqpumulum huunluornıu untuunıu tiup, np utin htennuuting uupunútig

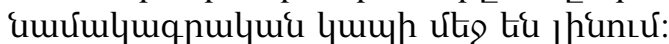

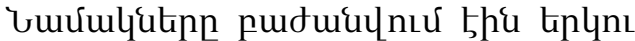
bứph. unughun, ulumunthnptiu hһц wu-

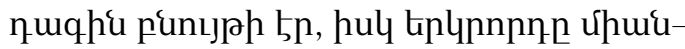
quưuuju unnn 2 , huuntumgnn, hupntuun

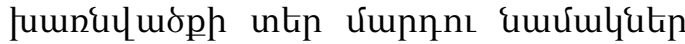
5hu:

ঢhpnın hưưunju úp2u fununıu $5 n$ unnưumpnluwiug úmuhu, nnnup uw'un-

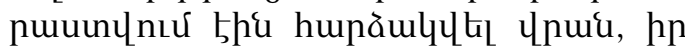

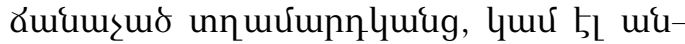
¿uiunp:

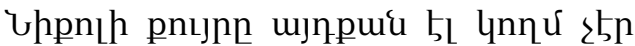

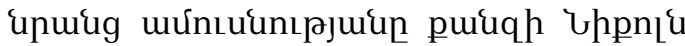
zwu unn niutip, hul Thpn puhuiumsh nnnh 5n l poh2l: Fujg ujnıu lnnưhg nu àtruuunn qnpounp $5 n$, puiqh um guuyuuntu th hp pnne lnnphu úp bưuưu-

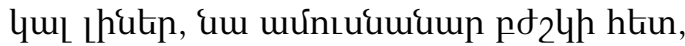
ny lluumurke unwu: Ujnutiu bl tnuy:

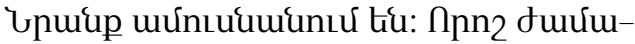

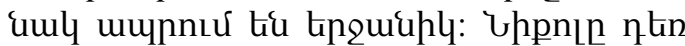

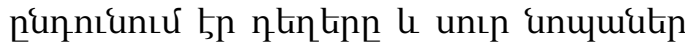

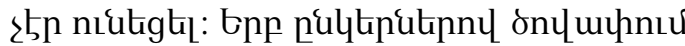
tú ghunıu, htinulhg ulumunnıu tiu úp lưne l. úp ungluw' nnquumphi le hn únne:

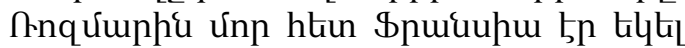

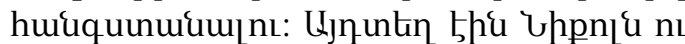
hptiug 2nquumunnn umpnhl: Uulquju ujn uwhhi Rujцtinutinu hptughg utinqujuguntu thu úp uúpnne nuulumph ununupha 54 nınıghnu qunqugúuru ququpsumblin, ujumbu nn úmplquigg út-

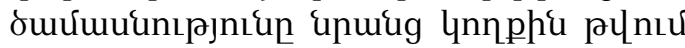
5n lnngunuu thu hptúg nhppu: ơưnpu-

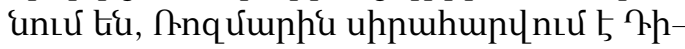
phi: Uın dưumuml Rppn ntin uto wq-

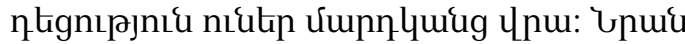

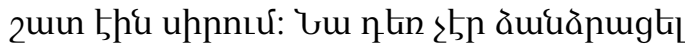

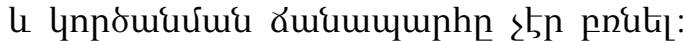
Uúpnne unuphuteph nupugpnư uu lưne hưump pugh uứnıuhe thutintg, thunıu

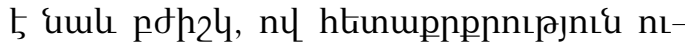

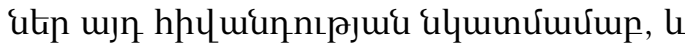

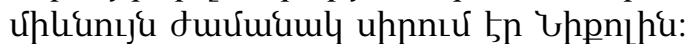

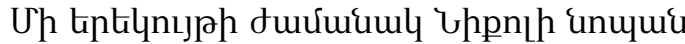

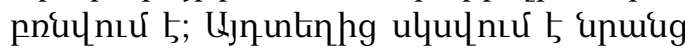
hupuptipnıpjnıkutiph fumbuunnıún' puup np unnteu nnqưunhe $5 n$ hujunuy tal:

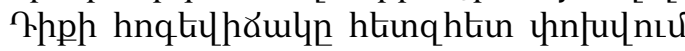




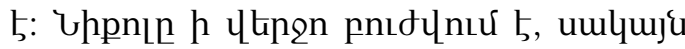

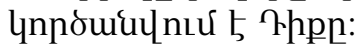

กnqu úunhu tiphunuumpr 5n' 18 unupt-

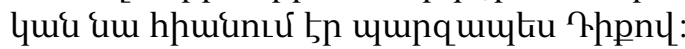

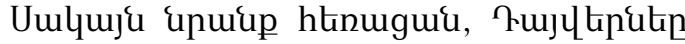
hpkiug pumlyuunu ljuipny thu wünnu: Thp \$puugh htiu npn2nuर 5 hhц wunu-

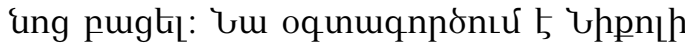

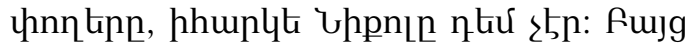

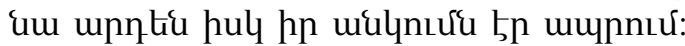

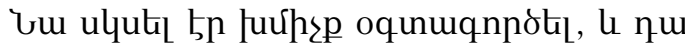

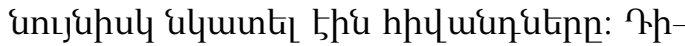

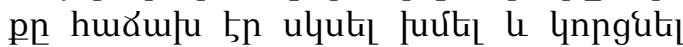
hpkiu: bl úp ưquư unupup uunkihu,

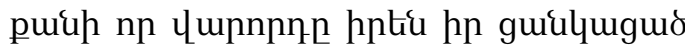

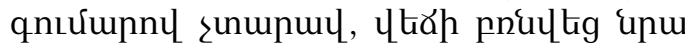

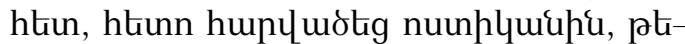
ukiun ¿qhunkn, nn uw nuonhlywu $5 \mathrm{l}$ ujn-

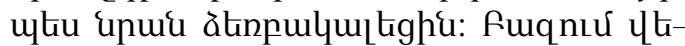
бtinhg lu pu2p2nıputinh htinn fluphe wquintig Rhphi:

flusphu qnhnı́umlynıрјư uju qqu-

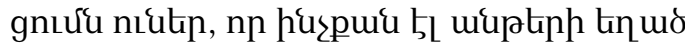

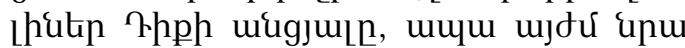

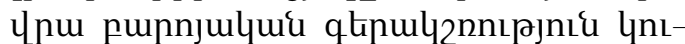

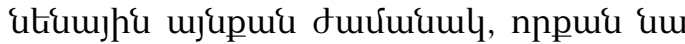
ukinp yqun hptiug:

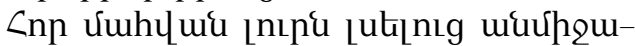

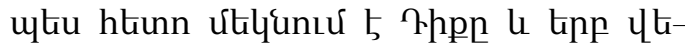

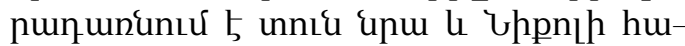

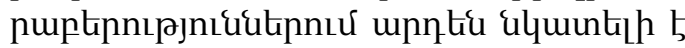
пununıu uununıpjnıun:

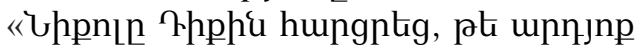

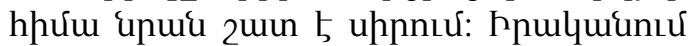

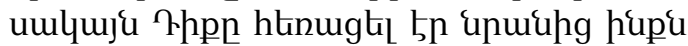

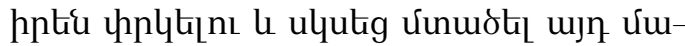
uhu: 'vu lnngntil $5 n$ hptiu' shưuum[nu,

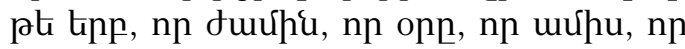

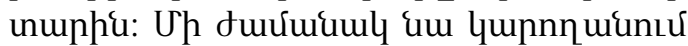

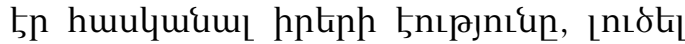

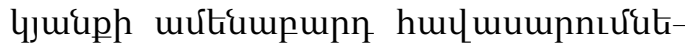

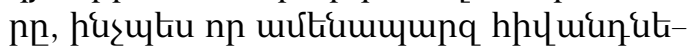

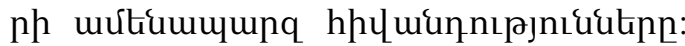

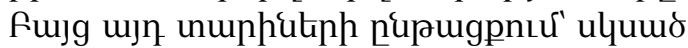
uhpnıhu 8jnıqtipuhh uhhe unughu wu-

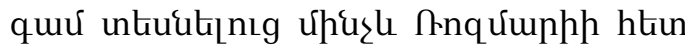

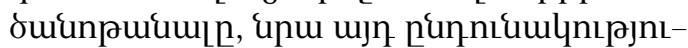

un uuunhouuumun prught 5 »» (U. \$hg2tipuln, 2015, 300):

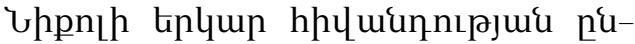
pugpnúu' ns hpplu pdh24, wJ hpplu ưunn, uugutiny whuц nn aquuduútinh úpøny,

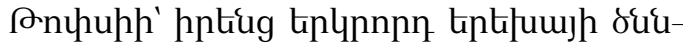
nhg htinn, rhpu qnuntiưurtal 5n' ut-

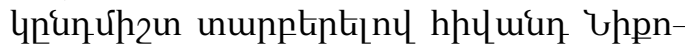
thu unnne Ghpnıhg: Uulquju unult

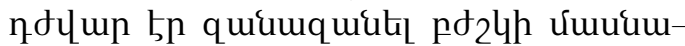
qhunulumu ounupugnıúp hp ununnıu owquo unp uununıpjntuhg: bpp unwøu-

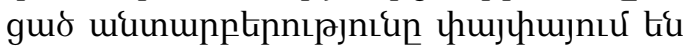

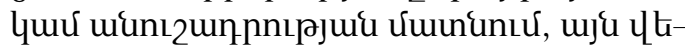

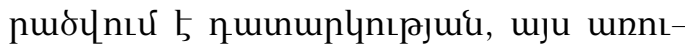

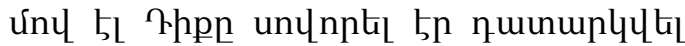

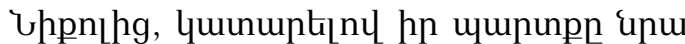
huuntey quúphi hulqunul, urqquagunıup nı wulhpp:

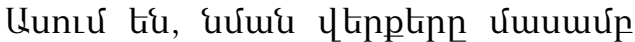

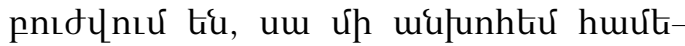

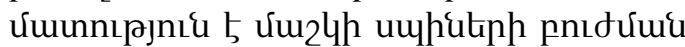

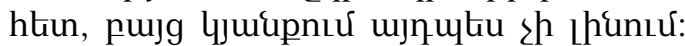

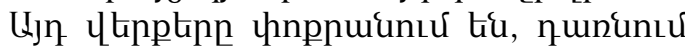
qunuutinh qiluplyh suih no tipptip steu

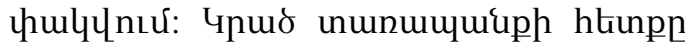

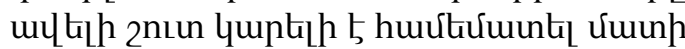
quu urph ynnuunh htiu: 9nıgt ununцm uthe util uuquu hh2tup ujn, pusg tipt

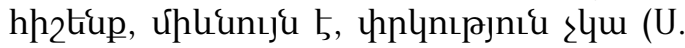
Shgetinuin 2015, 332):

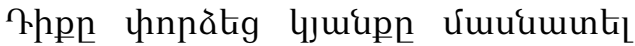

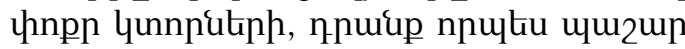

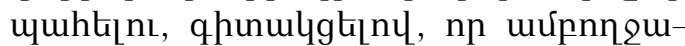

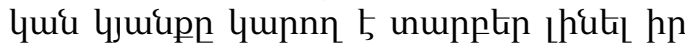
hul úuukphg, le, pugh ujn, punwuntup únu ưunnn humupulnn sh quínıu

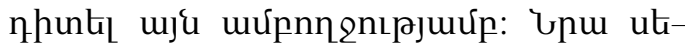

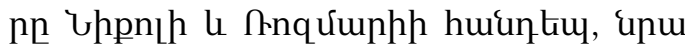

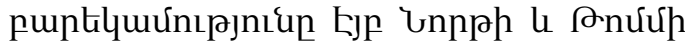

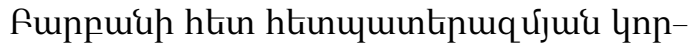

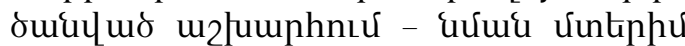
hunuptipnıpjnıuutinh úte úunnhl uju-

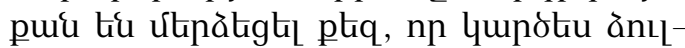

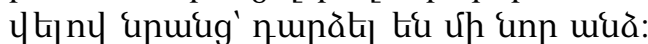

Thpu unnté tiplun durumuml 5 , hur

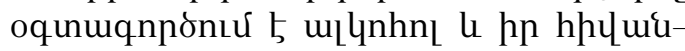




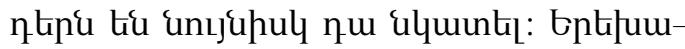

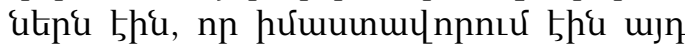

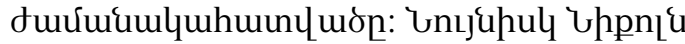

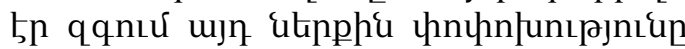

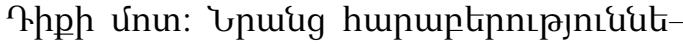
nnıu uwn hnuwupd $5 n$ únıunp qnpots $\mathrm{l}$

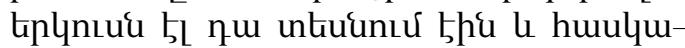
unıu:

-Ujuuku 2unntumlit sh thup,- uluktg

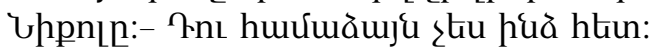

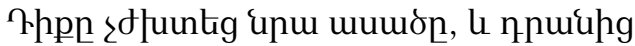

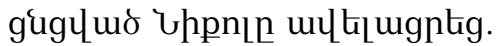

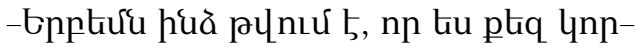
suitigh:

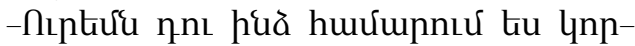

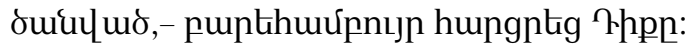

- bu nu ulquinh sniutih: fujg unuq

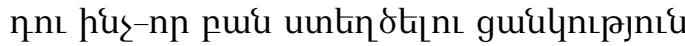
nıtuihn, hul hpưu' ynnowutint (U. \$hg¿tinum (1, 2015, 390):

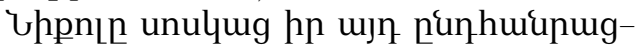
unn pudumpunulyud funuptinhg, pujg

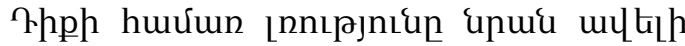

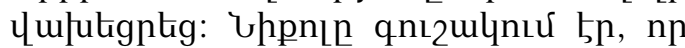

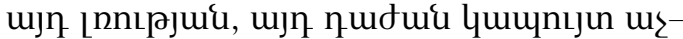
ptiph, tiptumutiph huintiu ujn wiun-

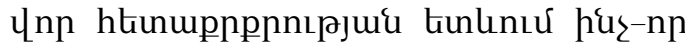

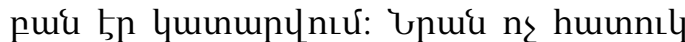

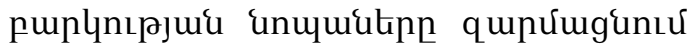
5hu Uhpnihu: Rppn huulume tiplum

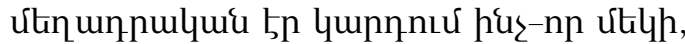

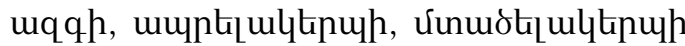

huugthe: Gunothu unu utrnu2lumphnıu wuhuulumug funnlp qun, nph úmuhu qunkilh 5n qni2ultal úhuja mú dưư-

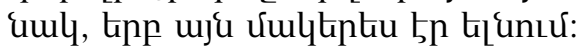

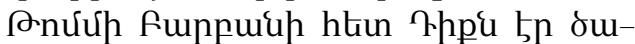
unpugntil uppnthu: bl qunotiu unuiug

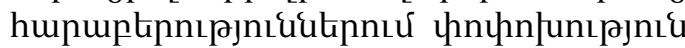

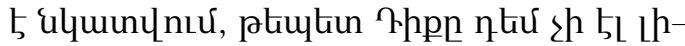

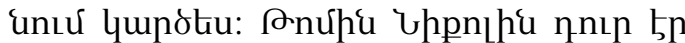
quihu: fuug úp zupup htionn Uhpnın

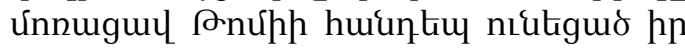

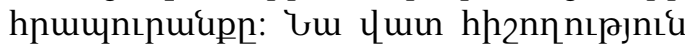

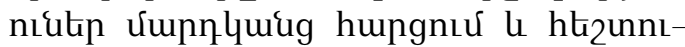

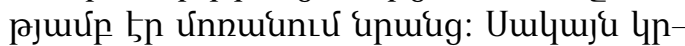

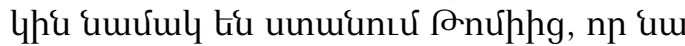

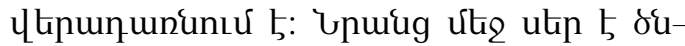

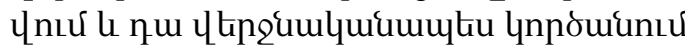
5 rusytunuteph ứnıuunıpjnıun, unuiug

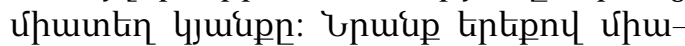
uhu unơunuunıu q nnıgnıu tiu: कrnúhu

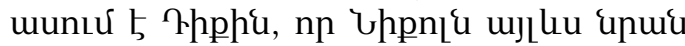

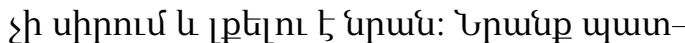

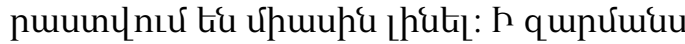

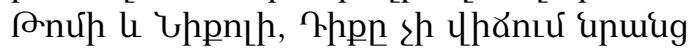

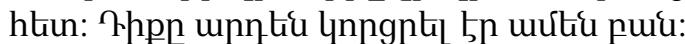

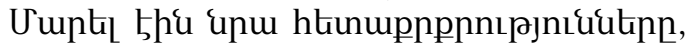
qqugúnıupuknn, ujuupp huuntiu nıutguo huornıpp: nnquumhe bL unu lnn-

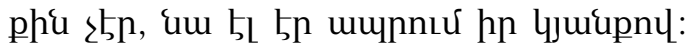

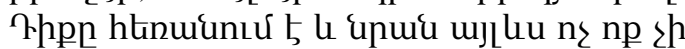

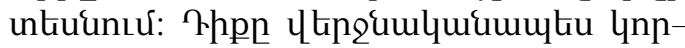

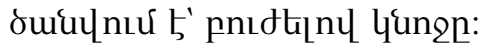

\section{4คULUนกrค3กrb}

1. James Truslow Adams, The Epic of America, New York,1931

2. Tender Is the Night by John Chamberlain, New York, 1934

3. Роман Лысцев, Статья по роману Ночь Нежна Ф. С. Фицджеральда, 2008

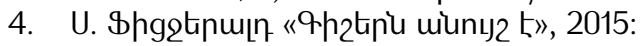


ИДЕЯ АМЕРИКАНСКОЙ МЕЧТЫ В РОМАНЕ С. ФИЦДЖЕРАЛЬДА “НОЧЬ НЕЖНА"

\title{
АНАИТ ШАХМУРАДЯН
}

Доцент факультета романо-германской

филологии, кандидат филологических наук

\begin{abstract}
АННА АРУТЮНЯН
Аспирант отделения итальянского языка и литературы факультета романо-германской филологии
\end{abstract}

Произведение «Ночь нежна» С. Фицджеральда является одной из самых мощных и глубоких его работ. Своего героя Дика автор представляет читателю талантливым, умным и полным духовного богатства, который, однако, духовно деградировал. Женившись на представительнице "очень богатого мира", Дик испытывает упадок духовного мира. Трагедия доктора личная и одновременно социальная. Это обвинение «очень богатым» и вердикт для буржуазной Америки, которая дала им власть и сделала их неприкосновенными.

\section{IDEA OF THE AMERICAN DREAM IN F. SCOTT FITZGERALD'S NOVEL “TENDER IS THE NIGHT"}

ANAHIT SHAHMURADYAN

PhD, Associate Professor of the Romance Germanic Philology Faculty, YSU

\section{ANNA HARUTYUNYAN}

Post graduate Student of the Spanish Language and Literature Division, Romance Germanic Faculty, YSU

The novel "Tender Is the Night" is one of the most prominent works of F. Scott Fitzgerald. He described his hero Dick as a smart, talented and a person full of spiritual richness, but who was unable to prevent himself from moral degradation. Doctor's tragedy is not only personal, but also social. The American son of a clergyman who is on the way to becoming a renowned psychologist when he falls in love with Nicole and marries her. Dick is extraordinarily charismatic and graceful at the start of the novel, but eventually falls to his ruin. 